\title{
Accelerated guided atomic pulse
}

\author{
S. Dyrting, ${ }^{1,2}$ Weiping Zhang, ${ }^{1}$ and B. C. Sanders ${ }^{1}$ \\ ${ }^{1}$ School of Mathematics, Physics, Computing and Electronics, Macquarie University, Sydney, New South Wales 2109, Australia \\ ${ }^{2}$ Department of Physics, The Hong University of Science and Technology, Clear Water Bay, Kowloon, Hong Kong \\ and Department of Physics, The University of Queensland, St. Lucia, Queensland 4072, Australia
}

(Received 29 January 1997)

\begin{abstract}
The deleterious effects of dispersion on a propagating coherent atomic pulse, along the axis of a travelingwave laser beam, can be ameliorated by the nonlinear self-interacting force due to dipole-dipole coupling between atoms. We show that a wide atomic pulse with a particular profile can retain its shape during propagation and, moreover, the momentum of the pulse increases due to photon absorption. For the wide soliton case, we demonstrate analytically that the self-interacting atomic force scales inversely with the third power of the pulse width. [S1050-2947(97)03009-6]

PACS number(s): 03.75.Fi, 42.50.Vk, 32.80.Lg
\end{abstract}

\section{INTRODUCTION}

Experimental success with generating Bose-Einstein condensates of atoms [1] has motivated theoretical studies of atom lasers, that is, sources of coherent atomic beams [2]. One such direction is the creation of coherent atomic pulses such as that achieved by rf output couplers for Bose-Einstein condensed atoms in a magnetic trap [3]. Unfortunately, localized atomic beams become decohered due to non-neglible dispersion and repulsive interatomic interaction during propagation. However, there exists a nonlinear interaction between atoms in a pulse due to the light-induced dipoledipole interaction between atoms [4,5]. A treatment of the propagation of a pulse, with an initial soliton profile, shows that a pulse can propagate without dispersion under appropriate conditions [6]. This treatment assumes that the nonlinear long-range dipole-dipole force can be treated as a point force, i.e., a Dirac $\delta$ function yielding a $\delta$ Bose-Einstein gas [7].

The dipole-dipole force produces an effective nonlinear interaction between atoms in the pulse that offsets dispersion and can lead to solitonlike behavior. The existence of a genuine soliton occurs only if the force is highly localized [6], but we show that solitonlike behavior is possible for the longrange force. We show that a wide pulse with an initial soliton profile does propagate with minimal dispersive effects and retains its longitudinal profile. The equations are solved numerically and, for a wide pulse, analytical expressions are also obtained. In particular the self-interaction force in the pulse is shown to scale inversely with the third power of the pulse width. We also show that the self-interaction force causes an acceleration of the pulse analogous to the Ramaninduced frequency shift in optical solitons [8].

\section{EQUATION OF MOTION}

The coherent atomic pulse propagates longitudinally along the waist of a traveling-wave laser beam where the waist is much wider than the transverse width of the atomic pulse. Nonlinear stochastic Schrödinger equations describe the dynamics of ground- and excited-state wave packets. For large detuning adiabatic elimination of the excited-state com- ponent allows the vector atomic field to be treated as a scalar field. With position designated by $x$, measured in units of $1 / k$ for $k=2 \pi / \lambda$ the wave number of the optical field, and time designated by $t$, measured in units of recoil time $\tau_{r} \equiv 1 / \omega_{r}$ for $\hbar \omega_{r}=\hbar^{2} k^{2} / 2 m$ the single-photon recoil energy, the equation of motion for the ground-state atomic wavepacket in one dimension is [6]

$$
i \frac{\partial \phi(x)}{\partial t}=-\frac{\partial^{2} \phi(x)}{\partial x^{2}}+N \int_{-\infty}^{\infty} d x^{\prime} Q\left(x-x^{\prime}\right)\left|\phi\left(x^{\prime}\right)\right|^{2} \phi(x),
$$

where $N$ is the total number of atoms in the condensate pulse and the kernel

$$
Q(x)=\kappa_{0} \delta(x)+\kappa_{1} D(x)
$$

is the effective two-body potential. The coefficient

$$
\kappa_{0}=4 \pi \beta k a
$$

is the strength of the dressed short-range interatomic interaction in the presence of the laser beam; $\beta$ is the average value of the mode density function transverse to the laser beam propagation axis; $a$ is the dressed $s$-wave scattering length; $D(x)$ is the long-range dipole-dipole potential; and

$$
\kappa_{1}=\frac{\gamma}{\omega_{r}} \frac{|\Omega|^{2}}{2 \Delta^{2}} \eta
$$

is the strength of the long-range interaction with $\gamma$ the linewidth of the atomic transition, $\Omega$ the Rabi frequency, $\Delta$ the detuning of the laser from the atomic transition, and

$$
\eta=\frac{1-i \gamma / 2 \Delta}{\left(1+\gamma^{2} / 4 \Delta^{2}\right)^{2}}
$$

The detuning is assumed to be sufficiently large such that $\eta=1$ and multiplicative stochastic terms can be ignored here [9]. 
Under appropriate conditions [4-6] the dipole approximation yields the one-dimensional long-range dipole-dipole potential in a traveling-wave laser beam

$$
D(x)=w(x) \cos (x)-k(x) \sin (x)
$$

for

$$
\begin{gathered}
w(x)=2 \pi \int_{0}^{\infty} d r r P(r) W(x, r), \\
k(x)=2 \pi \int_{0}^{\infty} d r r P(r) K(x, r),
\end{gathered}
$$

and

$$
P(r)=\frac{1}{2 \pi \sigma} e^{-r^{2} / 2 \sigma}
$$

the distribution function for the transverse laser mode. The functions $W(x, r)$ and $K(x, r)$ are the imaginary and real parts, respectively, of the nonlinear two-body correlation coefficient [Eq. (10) in Ref. [9]] averaged over orientations perpendicular to the laser axis

$$
\begin{gathered}
W(x, r)=\frac{3}{4}\left[-\sin ^{2} \theta \frac{\cos \xi}{\xi}+\left(1-3 \cos ^{2} \theta\right)\left(\frac{\sin \xi}{\xi^{2}}+\frac{\cos \xi}{\xi^{3}}\right)\right], \\
K(x, r)=\frac{3}{4}\left[\sin ^{2} \theta \frac{\sin \xi}{\xi}+\left(1-3 \cos ^{2} \theta\right)\left(\frac{\cos \xi}{\xi^{2}}-\frac{\sin \xi}{\xi^{3}}\right)\right],
\end{gathered}
$$

where we have assumed that the dipole moment for the coherent atomic pulse is aligned to the laser polarization such that $\cos ^{2} \theta=r^{2} / \xi^{2}$ and

$$
\xi^{2}=x^{2}+2 r^{2}+b^{2} .
$$

Expression (2.10) describes the potential for a scale large compared to the atomic size parameter $b$, chosen here to be 1 $\mathrm{nm}$, or $b=0.01$. The short-range interaction due to overlapping of internal electron states within the atom dominates on the length scale smaller than $b$ and the $\kappa_{0} \delta(x)$ term in Eq. (2.2) describes the potential on this length scale. Here we treat the case of attractive short-range interactions $\left(\kappa_{0}<0\right)$.

\section{THE SOLITON PROFILE}

For $\kappa_{0}<0$ and $\kappa_{1}=0$, Eq. (2.1) yields the soliton solution

$$
\Phi(x, t)=\phi(x) \exp \left(i \pi^{2} t / 2 x_{N}\right),
$$

with

$$
\phi(x)=\sqrt{\frac{\pi}{4 x_{N}}} \operatorname{sech}\left(\pi x / 2 x_{N}\right)
$$

and

$$
x_{N}=-\frac{2 \pi}{\kappa_{0} N}
$$

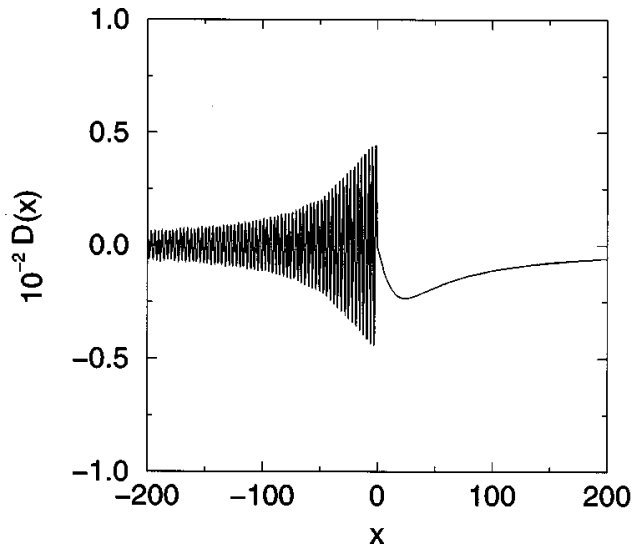

FIG. 1. Asymmetric one-dimensional long-range light-induced dipole-dipole potential $D(x)$ due to a traveling-wave laser beam as a function of the distance $x$ between two atoms.

The spatial distribution $|\Phi(x, t)|^{2}$ is time independent and can be measured directly, for example, by absorption imaging [1]. Once this function is known the position statistics can be calculated; in particular for the soliton we find $x_{\mathrm{rms}}=x_{N} / \sqrt{3}$ for the root-mean-square position. Therefore, as the number of atoms increases the soliton width becomes smaller. The Fourier transform of $\phi(x)$ gives the condensate wave function in the momentum representation

$$
\widetilde{\phi}(p)=\frac{1}{\sqrt{2 \pi}} \int_{-\infty}^{\infty} d x \phi(x) \exp (-i p x) .
$$

The wave function of the soliton in the momentum representation is

$$
\widetilde{\phi}(p, t)=\sqrt{\frac{x_{N}}{2}} \operatorname{sech}\left(x_{N} p\right) \exp \left(i \pi^{2} t / 2 x_{N}\right) .
$$

For low densities the momentum distribution function $|\widetilde{\phi}(p)|^{2}$ can be measured by absorption imaging after a period of ballistic expansion [10]. Such time-of-flight images can still be used to measure the mean momentum

$$
\langle p\rangle=\int_{-\infty}^{\infty} d p \quad p|\widetilde{\phi}(p)|^{2},
$$

which is unaffected by atomic collisions in the absence of external fields. For the soliton solution (3.1) $\langle p\rangle=0$. We would now like to know to what extent this picture of atomic soliton propagation is affected by the presence of the longrange dipole-dipole potential $D(x)$.

\section{ATOMIC PULSE DYNAMICS}

The effects of the long-range dipole-dipole potential are considered for a transverse mode with a width of approximately $1 \mu \mathrm{m}$ and where the laser is propagating in the positive $x$ direction. The first point to note is that this lightinduced dipole-dipole potential, depicted in Fig. 1, is not symmetric as it is generated by a traveling-wave laser beam with a specified direction. This asymmetry is due to the presence of a preferred direction defined by the direction of the laser. Second, $D(x)$ oscillates rapidly, on the scale of $\lambda$, for 

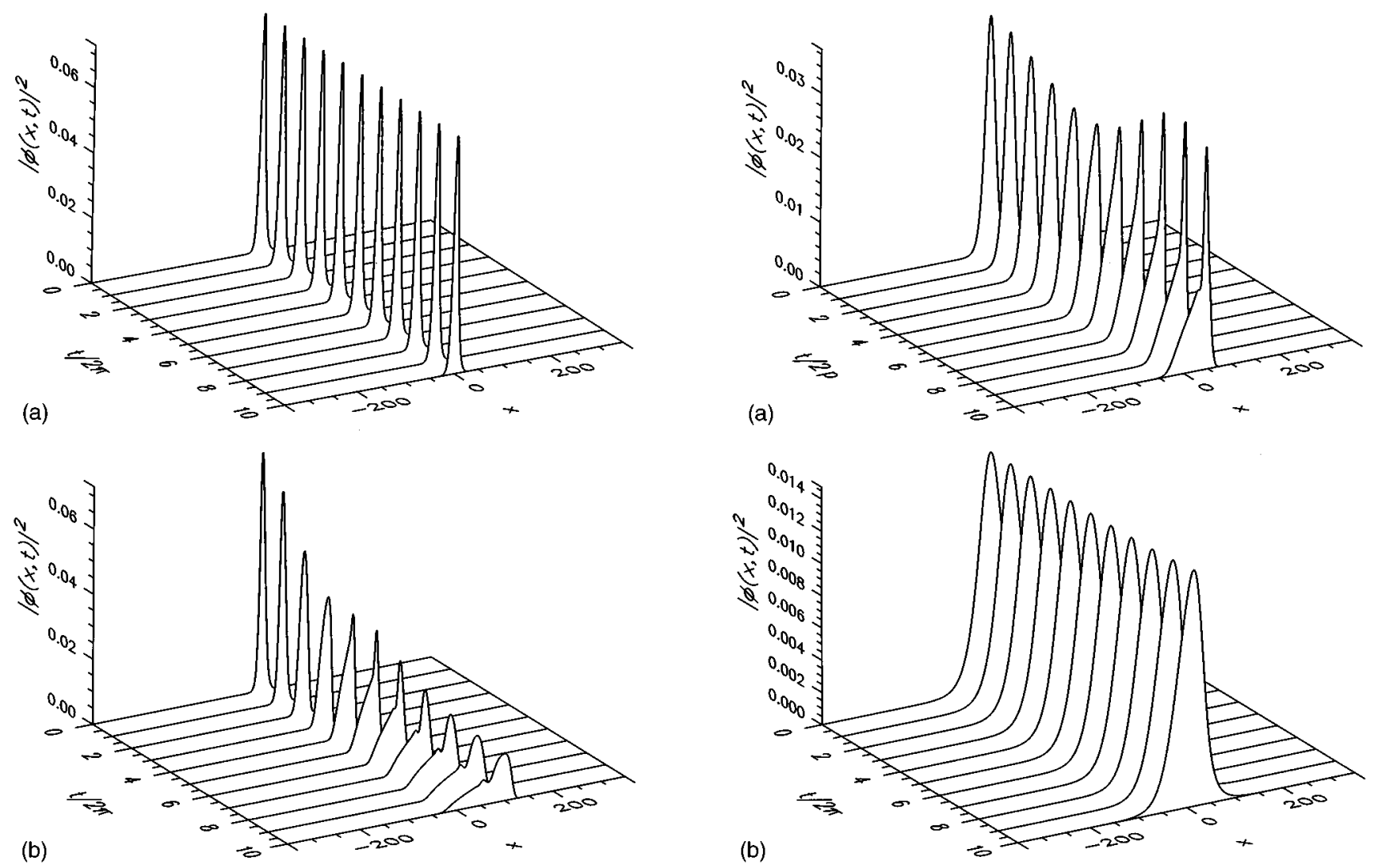

FIG. 2. Position distribution for coherent atomic pulse with negative $s$-wave scattering and $|\Omega| / \Delta=0.002, \lambda / a=-8521$, $\kappa_{0}=-5.8 \times 10^{-5}$, and $N=10000$. The two cases treated are (a) $\kappa_{1}=0$ and (b) $\kappa_{1}=5.17 \times 10^{-3}$.

$x<0$. Typical atomic condensates are wide and vary slowly on this scale; hence the potential experienced by the condensate can be approximated by the average potential achieved by setting $D(x)=0$ for $x<0$. This simplification yields an atomic pulse dynamics that is analogous to that considered by Gordon in his theory of optical soliton propagation in optical fibers with a time-asymmetric delayed response function [8]. For $x>0, D(x)$ decays slowly. Using Eqs. (2.6)(2.12), it can be shown that the potential has the asymptotic behavior

$$
D(x) \sim-\frac{3}{4} x^{-1}, \quad x \rightarrow+\infty .
$$

This indicates that the dipole interaction has a long range.

In solving Eq. (2.1) we treat the case of high laser detuning, weak laser field strength, and small negative $s$-wave scattering length. Under these conditions the loss of atoms and multiplicative stochastic fluctuations due to incoherent photon scattering can be neglected. The value of the $s$-wave scattering coefficient applies within the laser field and is different from the scattering length in the absence of the optical field. In Fig. 2 the evolution of the position distribution, or profile, for the atomic pulse prepared in the state (3.1), with $x_{N} \approx 11$, is plotted over ten recoil periods. For $\kappa_{1}=0$, in Fig. 2 (a), the pulse envelope is a soliton solution for the equation,

and the profile is unchanged over the time scale of propagation as expected. The profile changes noticeably, however, for $\kappa_{1}=5.17 \times 10^{-3}$ in Fig. 2(b). The pulse broadens and the center-of-mass position is shifted in the direction of laserbeam propagation due to the asymmetry of the long-range profile $D(x)$. The time scale for change in the profile and in mean position is the recoil time $\tau_{r}$, as expected from the proportionality of the nonlinear coefficient (2.4) and $\tau_{r}$.

The deterioration of the pulse profile can be reduced by modifying the density of the pulse, which in turn reduces the strength of the nonlinearity. The nonlinearity depends both on the coefficient of nonlinearity $\kappa_{1}$ and the magnitude of $N D\left(x-x^{\prime}\right)$ integrated against the density of the soliton. A reduction in the number of atoms $N$ in the pulse or, equivalently, an increase in the pulse width $x_{N} \propto 1 / N$ reduces the magnitude of this integral and the preservation of profile is improved during propagation. In Fig. 3 the position distribution for the pulse is given for the same parameters as for Fig. 2(b), except with reduced values of the total atomic number $N$. These figures illustrate the better preservation of the profile for pulses with reduced density.

Converting the equation of motion for the wave packet to momentum space provides an alternative interpretation for the improved propagation characteristics as well as revealing an acceleration of the pulse. The equation of motion (2.1) can be written as 


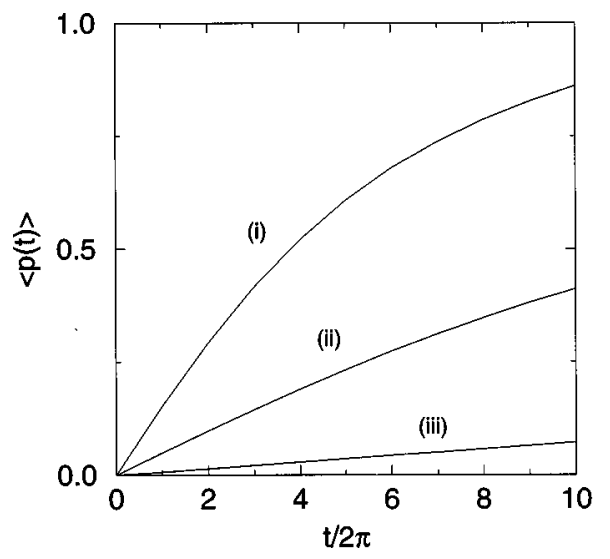

FIG. 4. Mean momentum of atomic pulse as a function of time with negative $s$-wave scattering and $|\Omega| / \Delta=0.002, \lambda / a=-8521$, $\kappa_{0}=-5.8 \times 10^{-5}$, and $\kappa_{1}=5.17 \times 10^{-3}$. The three cases treated are (i) $N=10000$, (ii) $N=5000$, and (iii) $N=2000$.

$$
\begin{aligned}
i \frac{d \widetilde{\phi}(p)}{d t}= & p^{2} \widetilde{\phi}(p)+\frac{N}{\sqrt{2 \pi}} \int_{-\infty}^{\infty} d p^{\prime \prime} \widetilde{Q}\left(p^{\prime \prime}\right) \widetilde{\phi}\left(p-p^{\prime \prime}\right) \\
& \times \int_{-\infty}^{\infty} d p^{\prime} \widetilde{\phi}\left(p^{\prime}+p^{\prime \prime}\right) \widetilde{\phi}^{*}\left(p^{\prime}\right),
\end{aligned}
$$

with $\widetilde{Q}(p)$ the Fourier transform of $Q(x)$. The mean momentum $\langle p(t)\rangle$ is readily calculated and plotted in Fig. 4 for the parameters considered in Figs. 2 and 3. These figures show a continuous increase in the mean momentum, which is particularly pronounced for large numbers of atoms (narrow pulses). This self-force is analogous to the self-frequency shift of an optical soliton propagating in an optical fiber [9]. The optical pulse undergoes a continuous downshift in frequency due to Raman effects. The acceleration of the atomic pulse is analogous to the frequency shift of the optical pulse, except that, in the atomic case, the momentum, or velocity, of the pulse is altered by the nonlinear interaction between the atoms and photons in the traveling-wave laser beam. The direction of motion in the laser beam determines the direction of acceleration of the atomic pulse.

For short times the mean momentum increases linearly. Thus we define the self-force as the initial rate of change

$$
F=\left.\frac{d\langle p\rangle}{d t}\right|_{t=0} .
$$

Substituting the pulse momentum distribution (3.5) into the right-hand side of Eq. (4.2) gives the self-force

$$
F=\sqrt{2 \pi} x_{N}^{-3} \frac{\kappa_{1}}{\kappa_{0}} \int_{-\infty}^{\infty} d z z^{3} \frac{\widetilde{D_{a}}\left(z / x_{N}\right)}{\sinh ^{2} z}
$$

with $\widetilde{D_{a}}(p)$ the Fourier transform of the antisymmetric part of Eq. (2.6),

$$
\widetilde{D_{a}}(p)=\frac{1}{\sqrt{2 \pi}} \int_{0}^{\infty} d x \sin (p x) D(x)
$$

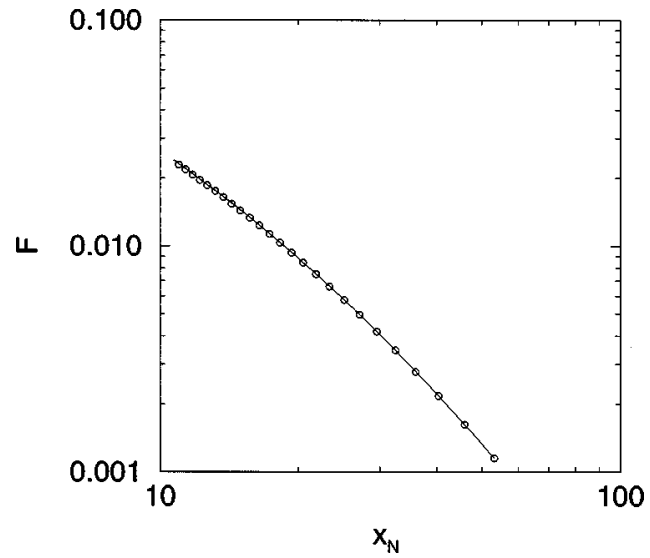

FIG. 5. A $\log -\log$ plot of the self-force $F$ (solid), from Eq. (4.5), vs. the tangent of $\langle p(t)\rangle$ at $t=0$ (circles) from Fig. 4.

For large $x_{N}$ (hence small $N$ ) Eq. (4.5) suggests the asymptotic relation $F \propto x_{N}^{-3}$. This scaling law is similar to Gordon's result for the soliton self-frequency shift in an optical fiber [9], except that here the falloff of the force is weaker because of the long range of the dipole-dipole interaction as embodied by Eq. (4.1). The agreement between the numerical solution of $\langle p(t)\rangle$ and the asymptotic analytical solution is excellent, as shown in Fig. 5. The large decrease in the self-force with increasing atomic pulse width accounts for the stability of propagation of the lower-density atomic soliton where $N$ is sufficiently small (and hence $x_{N}$ is sufficiently large). An initial atomic pulse shape for which the envelope is a soliton shape in the case that $\kappa_{1}=0$ will retain its shape on the time scale $\tau_{r}$, the recoil time, for coherent atomic pulses that are wider than the optical wavelength of the guiding laser beam.

\section{CONCLUSION}

In summary, dispersion limits the efficacy of propagating coherent atomic pulses, but the nonlinear light-induced dipole-dipole force between atoms can be exploited to reduce the effects of dispersion. The nonlinearity results in a pulse compression that opposes the spreading of the pulse due to dispersion. Under conditions where deviations from soliton behavior are perturbative, the pulse profile remains intact on the time scale of the recoil time $\tau_{r}$. Negative scattering lengths have been treated here, although the formalism permits both negative and positive scattering lengths to be treated. The negative scattering length offers advantages over the positive scattering length insofar as a consistent pulse profile during propagation is concerned.

In addition to the importance of preserving the coherence and longitudinal profile of the propagating atomic pulse, the nonlinear long-range light-induced dipole-dipole force is significant as a pulse accelerator. The mass of the atomic pulse causes it to be accelerated by gravitational forces. A lightinduced atomic waveguide is thus important in this context as a mechanism for reducing or negating the effects of grav ity. An application of this waveguide could be to maintain the location of the pulse over some period of time or even to lift the pulse against the pull of gravity.

We have assumed that the laser field detuning $\Delta$ is suffi- 
ciently large that loss and multiplicative noise terms can be ignored from the dynamics of the atomic pulse. However, a complete simulation of the pulse dynamics would include the loss and noise terms, and this work is in progress.

\section{ACKNOWLEDGMENTS}

This work has been supported by the Australian Research Council and by a Macquarie University Research Grant.
[1] M. Anderson, J. R. Ensher, M. R. Matthews, C. E. Wieman, and E. A. Cornell, Science 269, 198 (1995); C. C. Bradley, C. A. Sackett, J. J. Tollet, and R. Hulet, Phys. Rev. Lett. 75, 1687 (1995); K. B. Davis, M.-O. Mewes, M. R. Andrews, N. J. van Druten, D. S. Durfee, D. M. Kurn, and W. Ketterle, ibid. 75, 3969 (1995); M.-O. Mewes, M. R. Andrews, N. J. van Druten, D. M. Kurn, D. S. Durfee, C. G. Townsend, and W. Ketterle, ibid. 77, 416 (1996); D. S. Jin, J. R. Ensher, M. R. Matthews, C. E. Wieman, and E. A. Cornell, ibid. 77, 420 (1996); M.-O. Mewes, M. R. Andrews, N. J. van Druten, D. M. Kurn, D. S. Durfee, C. G. Townsend, and W. Ketterle, ibid. 77, 988 (1996).

[2] H. M. Wiseman and M. J. Collett, Phys. Lett. A 202, 246 (1995); R. J. C. Spreeuw, T. Pfau, U. Janicke, and M. Wilkens, Europhys. Lett. 32, 469 (1995); M. Olshanii, Y. Castin, and J. Dalibard, in Proceedings of the XIIth Conference on Lasers Spectroscopy, edited by M. Inguscio, M. Allegrini, and A. Sasso (World Scientific, Singapore, 1995); A. M. Guzmán, M. Moore, and P. Meystre, Phys. Rev. A 53, 977 (1996); H. M. Wiseman, A-M. Martins, and D. F. Walls, Quantum Semiclas- sic. Opt. 8, 737 (1996); M. Holland, K. Burnett, C. Gardiner, J. I. Cirac, and P. Zoller, Phys. Rev. A 54, R1757 (1996); G. M. Moy, J. J. Hope, and C. M. Savage (unpublished); H. M. Wiseman (unpublished).

[3] M.-O. Mewes, M. R. Andrews, D. M. Kurn, D. S. Durfee, C. G. Townsend, and W. Ketterle, Phys. Rev. Lett. 78, 582 (1997).

[4] W. Zhang and D. F. Walls, Quantum Opt. 5, 9 (1993); W. Zhang, Phys. Lett. A 176, 225 (1993).

[5] G. Lenz, P. Meystre and E. M. Wright, Phys. Rev. Lett. 71, 3271 (1993).

[6] W. Zhang, D. F. Walls, and B. C. Sanders, Phys. Rev. Lett. 72, 60 (1994).

[7] E. H. Lieb and W. Linger, Phys. Rev. 130, 1605 (1968); J. B. McGuire, J. Math. Phys. (N.Y.) 8, 662 (1964); C. N. Yang, Phys. Rev. Lett. 19, 1312 (1967).

[8] J. P. Gordon, Opt. Lett. 11, 663 (1986).

[9] W. Zhang and D. F. Walls, Phys. Rev. A 49, 3799 (1994).

[10] K. B. Davis, M.-O. Mewes, M. R. Andrews, N. J. van Druten, D. S. Durfee, D. M. Kurn, and W. Ketterle, Phys. Rev. Lett. 75, 3969 (1995). 